\title{
APRENDIZAGEM ATIVA DO CÁLCULO NA ENGENHARIA AUXILIADA POR SOFTWARE
}

João Carlos Cordeiro Barbirato-jccb@ctec.ufal.br

Maylla Guedes Cabral-maylla.gc@outlook.com

Maria Gabriella Lanverly de Melo Muniz-gabilmm@hotmail.com

Eduarda Abreu Vanderlei de Souza Silva-eduarda.silva@ctec.ufal.br

Universidade Federal de Alagoas, Centro de Tecnologia, Curso de Eng. Civil

Campus A. C. Simões - Av. Lourival de Melo Mota, S/N

57072-900 - Maceió - AL

Resumo: Os jovens do mundo atual são cem por cento conectados a algum tipo de rede social, com acessos infindos de dados e informações de maneira instantânea e em qualquer lugar. A chegada no ensino superior traz expectativas de formas atualizadas de aprendizagem, na velocidade em que a geração costuma se movimentar. O choque nos primeiros períodos das engenharias nos quais as disciplinas de matemática, tão caras para a base dos cursos, são ministradas e, em grande parte, na maneira clássica de sala de aula. Isso tem levado a altos indices de reprovação e evasão. Existem, há tempos, metodologias mais interessantes para serem utilizadas inclusive nos ciclos básicos das engenharias. Neste contexto se insere o presente trabalho, abordando experiência da aprendizagem ativa em disciplinas de cálculo, partindo do modelo híbrido, auxiliado por plataforma informatizada. Estudantes de Engenharia Civil do Centro de Tecnologia da Universidade Federal de Alagoas foram protagonistas na aprendizagem ativa utilizando nas aulas extraclasse a plataforma MAPLE. As aulas tradicionais aconteceram e o desenvolvimento do estudo extraclasse foi pautado por simulações de questões, alterando parâmetros e observando a mudança no comportamento, além de melhor entendimento das funções e suas manipulações matemáticas. A associação com problemas efetivos de engenharia serviu de motivação ao aprendizado aprofundado da base de cálculo para o estudante do Curso. São apresentados três casos estudados no presente trabalho, sob a orientação de um professor engenheiro para o direcionamento à metodologia ativa e consolidação em encontros para definir estratégias e esclarecer entendimentos. As conclusões foram positivas, conforme pode ser visto no item correspondente do presente trabalho.

Palavras-chave: Aprendizagem Ativa, Maple, Cálculo Diferencial e Integral. 


\section{INTRODUÇÃO}

Os jovens do mundo atual são cem por cento (100\%) conectados a algum tipo de rede social, com acessos infindos de dados e informações de maneira instantânea e em qualquer lugar. Por outro lado, eles acreditam que o ensino superior é de extrema importância na construção do futuro tanto próprio quanto do País; mas querem mais praticidade, menos complicação nas atividades curriculares, algumas iguais há séculos. Portanto, buscam aulas mais interativas e tecnológicas, e que enfatizem trabalhos em grupos que se relacionem ao empreendedorismo e ao impacto social.

Nos cursos das ciências exatas, assim que o aluno ingressa nas universidades, depara-se com o chamado ciclo básico: cálculos, físicas, linguagens de programação, entre outros conhecimentos particionados e sequenciados na forma de disciplinas. Constata-se uma grande dificuldade por parte dos calouros (alunos ingressantes) para entender tais disciplinas, saber como estudá-las e como resolver questões referentes às mesmas. As ferramentas de aprendizado com metodologia própria, parecem não serem passadas no Ensino Médio. Por outro lado, a forma tradicional de ministração dessas disciplinas básicas, não tem permitido a inclusão de ferramentas computacionais ou ensaios de aulas ativas. Por isso, estima-se que as ferramentas computacionais são grandes aliadas nesse contexto, onde o estudante pode ter noção visual do comportamento de funções (algo extremamente importante) e a sua manipulação matemática. O sentido físico parece estar mais presente com gráficos explicativos (das funções, de suas derivadas, dos intervalos, dentre outros).

As experiências relatadas pelos pesquisadores em diversos trabalhos, demonstram que o modo tradicional do ensino da matemática no ensino superior não mais motiva a maioria dos estudantes. As chamadas metodologias ativas estão sendo aplicadas cada vez em maior escala como resposta ao modo tradicional de ensino. No entanto, verifica-se a resistência dos docentes mais tradicionais, bem como dos próprios discentes ao terem que se adaptar às novas metodologias. É o caso do ensino a partir das salas invertidas, no qual o estudante recebe a responsabilidade de estudar com antecedência os assuntos a serem abordados em sala de aula, deixando para esse momento a discussão rica sobre os mesmos, avaliação dos caminhos tomados, das interações feitas ou não, e mais (SACHETTI, 2017; JAAFAR e LIN, 2017; TING et al., 2019; KLEIN et al.; 2019; e ISTIANDARU et al.; 2019). Abrem-se espaços para dinâmicas na forma de jogos, envolvendo conteúdos "duros" de cálculo de forma mais "leve", pretendendo-se sempre a atenção do estudante, sua motivação para estar no centro das decisões de aprendizado, trabalhando ideias, cenários, pesquisas, dentre outros aspectos.

Ao longo do tempo pesquisadores relataram suas experiências com diversas ferramentas de ajuda ao aprendizado. Arango et al. (2015) apresentam práticas pedagógicas embasadas no uso de objetos de aprendizagem virtual para o aprendizado de cálculo diferencial. Aplica os conceitos, série de otimizações a partir do software livre GeoGebra. Vislumbra maior dinâmica na aprendizagem que permite ao estudante maior autonomia para análise de problemas práticos, dentro do curso envolvido no experimento. Em outra linha, Paula e Soares (2016) concentramse em proporcionar aos professores de cálculo ferramentas computacionais que, além de permitir a experimentação de metodologias ativas de aprendizagem, poderão ter relatórios diretos dos acertos e erros de cada estudante. Isso vai permitir, ainda em sala de aula, tomar medidas para conduzir os estudantes nas trilhas corretas para o adequado aprendizado.

Neste contexto se insere o presente trabalho, experimentando uma metodologia híbrida, complementando a tradicional disciplina em sala de aula de cálculos com tarefas extraclasse, auxiliadas por plataforma informatizada MAPLE, na qual o os estudantes são os protagonistas da aprendizagem. A chamada aprendizagem ativa é experimentada com estudantes de 
engenharia civil, desenvolvendo metodologia encaixada aos anseios deles próprios, com orientações periódicas de rumo e esclarecimentos da aplicação dos conteúdos estudados na prática da engenharia.

\section{METODOLOGIA EMPREGADA}

As disciplinas de cálculo do curso de engenharia civil do CTEC da UFAL são ministradas de maneira tradicional, por professores experientes do Instituto de Matemática, geralmente, utilizando-se quadro para as exposições e livros com teorias e bateria de exercícios para sedimentação dos conhecimentos. A forma clássica com que se apresentam os conhecimentos da matemática aos não matemáticos, estudantes de engenharia por exemplo, ao longo do tempo não tem se mostrado atraente à maioria dos estudantes. A motivação pretendida pelo professor não é atingida e o resultado tem sido frustrante para todos (discentes e docentes), com reprovações e evasões em excesso, apontam com propriedade Félix e Oliveira (2016). A inserção de manipulações outras do conteúdo ensinado, explorando as funções em suas representações gráficas, experimentando cenários variados, apropriação de problemas da engenharia, apresenta-se como alternativa para trazer o estudante para o centro do estudo, da pesquisa, do ensino aos colegas, ativamente atuando na aprendizagem.

Ressalte-se aqui a importância da base matemática para a formação dos engenheiros; disto não se abre mão.

Com estudantes matriculados nas disciplinas de cálculo I, primeiramente, e cálculo II, elaborou-se um plano de estudo a ser acompanhado por um professor tutor, dos setores da engenharia civil, visando aplicação dos conhecimentos matemáticos. O plano de metodológico consistiu em assistir as aulas tradicionais, com os responsáveis pelas disciplinas e trabalhar nas resoluções manualmente (como de praxe) e utilizando ferramentas computacionais, na linha da aprendizagem colaborativa.

$\mathrm{Na}$ linha tradicional, os estudantes estariam em contato com os conceitos, concatenação dos conhecimentos do ensino médio ao ensino superior, os caminhos utilizados para analisar um problema matematicamente, dentre outros aspectos.

Já na linha da aprendizagem colaborativa, as estudantes estariam em um ambiente automatizado, no qual poderiam definir o problema, selecionar as equações, variáveis, intervalos, dentre outros, e seguir na investigação mais completa. A começar pela facilidade de desenhar os gráficos correspondentes às funções, nos diversos intervalos (simulando vários cenários para entender o comportamento das funções), os gráficos de suas derivadas, por exemplo. Desta maneira, pretendia-se ter mais informações sobre o problema que se está resolvendo, de forma a melhor entender o problema, no sentido de dominar a maioria de suas formas e comportamentos. As operações matemáticas também poderiam ser facilmente executadas pela ferramenta computacional e, listando, se necessário, cada passo no desenvolvimento da solução. Quando se domina minimamente a ferramenta computacional, a construção de cenários mais completos com informações se torna uma realidade com o gasto de pouco tempo. Portanto, há como fazer variações da mesma questão e obter resultados, comparando seus comportamentos, de forma razoavelmente rápida. Essa experiência sedimenta o aprendizado dos conhecimentos e expõe o estudante a um número maior de aplicações do que no modo tradicional.

No presente trabalho foi utilizada a ferramenta computacional MAPLE, plataforma que permite a manipulação de expressões algébricas, simbólicas, e agrega a representação gráfica 2D e 3D de forma bem amigável ao usuário, permitindo a integração de funções, áreas e volumes com facilidade. 
Com o advento das Novas Diretrizes Curriculares das Engenharias, os projetos pedagógicos dos cursos devem proporcionar mudanças significativas, incluindo-se a incorporação de novas metodologias de ensino/aprendizado, nas quais as metodologias ativas são bastante adequadas e desejadas (OLIVEIRA, 2019).

\section{ESTUDO DE CASOS}

Para ilustrar a evolução dos estudos de cálculo utilizando-se ferramenta de aprendizagem colaborativa, serão apresentados três casos abordados, desenvolvidos seguindo as simulações computacionais, procurando responder aos quesitos do problema matemático bem como colhendo mais informações, no sentido de melhor dominar a aplicação. Nos três casos abordados, os estudantes tiveram as aulas nas salas tradicionais e partiram para os estudos extraclasse utilizando a plataforma informatizada para resolver os problemas indicados nas diversas seções dos livros adotados. Alguns encontros com o professor orientador foram feitos na ideia de levantar aplicações da engenharia que poderiam ser modeladas com os assuntos matemáticos que estavam sendo estudados. Com o auxílio da ferramenta computacional, os estudantes puderam explorar modificações várias nos parâmetros do problema, criando cenários para um espectro bem maior para o aprendizado. A partir daí, nos diversos espaços de estudos, os estudantes puderam, individualmente e em grupo, ser os protagonistas do estudo, regulando a aprendizagem com mais detalhes, com o entendimento mais amplo, com a sedimentação dos conhecimentos importante para o aprendizado.

\subsection{Teorema do valor médio}

Nesta aplicação, será abordado um problema de cálculo 1 correspondente ao Teorema do Valor Médio. A base teórica resumida é como se segue (Figura 1):

Figura 1 - Definição do problema matemático.

Seja f uma função que satisfaça as seguintes condições:

1. f é contínua no intervalo fechado $[a, b]$.

2. f é derivável no intervalo aberto $(\mathrm{a}, \mathrm{b})$.

Então, existe um número c em que $(\mathrm{a}, \mathrm{b})$ tal que

Ou, de maneira equivalente,

$$
f^{\prime}(c)=\frac{f(b)-f(a)}{b-a}
$$

$$
f(b)-f(a)=f^{\prime}(c)(b-a)
$$

Fonte: Os autores.

Para ilustrar o teorema do valor médio, considere-se a função Equação (1) e seu intervalo de análise.

$$
f(x)=x^{3}-x, a=0, b=2 .
$$

A concepção da análise, com o auxílio de ferramenta computacional e na linha do protagonismo do estudante (em metodologias ativas), parte-se da necessidade do conhecimento da função, estabelecendo seu gráfico em intervalo majorado (na forma de investigação pesquisa). Segue com a particularização ao intervalo definido, e o que mais é definido na conceituação do teorema de valor médio. A seguir, apresenta-se roteiro elaborado dentro da plataforma MAPLE (Figura 2), na qual se incluem textos explicativos e se realizam os passos 
C. COBENCE 2020

"Os desafios para formar hoje o engenheiro do amanhã"
$\mathrm{Ol} \mathrm{a} \mathrm{O3}$ de dezembro Evento On-line

algébricos, além da elaboração da representação gráfica das equações envolvidas nos intervalos pertinentes (Figura 3).

Figura 2 - Desenvolvimento do problema 3.1 no ambiente MAPLE, parte I: conhecendo a função.

A solução assitida é como se segue:

restart

$f:=x \rightarrow x^{3}-x=x \rightarrow x^{3}-x$

Para melhor conhecer a função, considera-se um intervalo maior.

$\operatorname{plot}(f(x), x=-2 . .3, y=-10 . .10)$

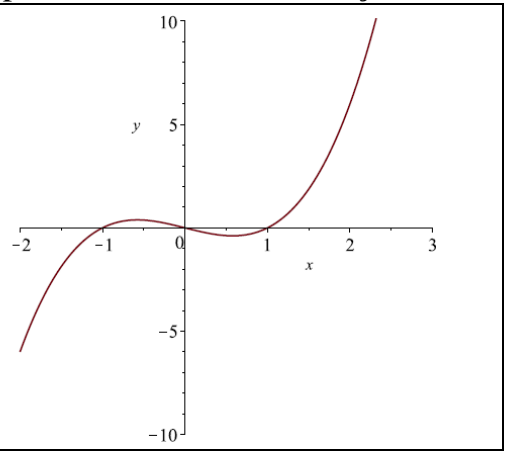

Fonte: Os autores.

Figura 3 - Desenvolvimento do problema 3.1 no ambiente $M A P L E$, parte II: respondendo à questão posta.

Considere, agora, o intervalo $[\mathrm{a}, \mathrm{b}]=[0,2]$ :

$a:=0=0$

$b:=2=2$

A função $f$ nos valores de a e b resulta em:

$f(a)=0$

$f(b)=6$

Já a primeira derivada de $f \dot{e}$ :

$c:=\operatorname{diff}(f(x), x)=3 x^{2}-1$

Uma vez que $f$ é uma função polinomial, então ela é continua e derivável para todo $\mathrm{x}$;

logo, é certamente é contínua em $[0,2]$ e derivável em $(0,2)$.

Portanto, pelo Teorema do Valor Médio, existe um número $\mathrm{x}$ em $(0,2)$ tal que:

$$
\begin{aligned}
& f(b)-f(a)=c \cdot(b-a)=6=6 x^{2}-2 \\
& s:=\operatorname{solve}\left(6=6 x^{2}-2, x\right)=-\frac{2}{3} \sqrt{3}, \frac{2}{3} \sqrt{3}
\end{aligned}
$$

Como o intervalo determinado é $[0,2] \log \mathrm{x}=-\frac{2}{3} \sqrt{3}$, que não o satisfaz, pois não está contido no intervalo; já $x=\frac{2}{3} \sqrt{3}$ pertence ao intervalo dado.

Então, a reta tangente em $\mathrm{x}=\frac{2}{3} \sqrt{3}$ é paralela à reta secante nos valores $0 \mathrm{e} 2$, conforme a seguir:

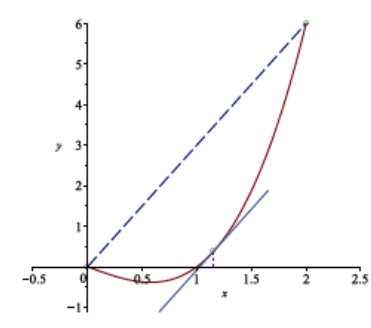

Fonte: Os autores.

\subsection{Derivadas e taxas de variação}

Nesta aplicação, será abordado um problema de cálculo 1 referente ao conceito de derivadas e taxas de variação. A ideia é interpretar de modo natural os limites, conceituais de derivadas, analisando a tendência da reta secante se transformar à tangente no ponto determinado (Figuras 5, 6 e 7). O enunciado do problema matemático é conforme a Figura 4. 
Figura 4 - Enunciado do problema 3.2 sobre derivadas e taxa de variação.

Pede-se encontrar uma equação da reta tangente à curva no ponto dado:

$f:=x \rightarrow 4 x-3 x^{2}$, no ponto $(2,-4)$

Fonte: Os autores

Figura 5 - Desenvolvimento do problema 3.2 no ambiente MAPLE, parte I:explorando os passos do limite.

Passos da resolução assistida (e ativa):

restart

$f:=x \rightarrow 4 x-3 x^{2}=x \rightarrow 4 x-3 x^{2}$

O ponto $(2,-4)$ pertence a curva dada. Logo,

pela definição de derivas, tem-se que:

$\lim _{h \rightarrow 0} \frac{f(a+h)-f(h)}{h} ;$ $\lim _{h \rightarrow 0} \frac{12+4 h-3(2+h)^{2}}{h}$

Tem-se que a $=2$, em que a é a abscissa do ponto dado, resulta em:

$m:=\lim _{h \rightarrow 0} \frac{f(2+h)-f(2)}{h}=-8$

$=\lim _{h \rightarrow 0}(-3 h-8) \quad[$ factor $]$

$=\lim _{h \rightarrow 0}-3 h+\lim _{h \rightarrow 0}(-8) \quad[$ sum $]$

$=\lim _{h \rightarrow 0}-3 h-8$

$=-3 \lim _{h \rightarrow 0} h-8$ constantmulti,

$=-8$

Passos da resolução do limite disponibilizados pela plataforma, de maneiras a esclarecer ao usuário: $\lim _{h \rightarrow 0} \frac{12+4 h-3(2+h)^{2}}{h}=-8$

Fonte: Os autores

Figura 6 - Desenvolvimento do problema 3.2 no ambiente MAPLE, parte II: determinação da reta tangente.

Portanto, a equação da reta tangente no ponto $(2,-4)$ e coeficiente angular $\mathrm{m}=$ -8 , é representada por:

$Y:=m \cdot(x-2)+(-4)=-8 x+12$

Pode-se, agora, fazer a representação gráfica das duas funções, a primitiva e a derivada.

Aqui, pode-se, de maneira criativa, trazer a movimentação conceitual da reta como secante,

passando para tangente ao ponto definido. Experiência interessante para sedimentar

conceitos.

$\operatorname{plot}([f(x), Y])$

Fonte: Os autores

A resolução clássica do problema em tela, neste ponto, encontra-se feita. Embora os limites foram passo a passo explicitados (a plataforma permite e ajuda bastante isso), ainda seria importante explorar a ideia gráfica do limite. O entendimento fica mais interessante; é o que se vê nas representações da Figura 7. Há como fazer uma sequência dinâmica desses gráficos, 
(C) COBENCE

"Os desafios para formar hoje o engenheiro do amanhã"
$\mathrm{Ol}$ a $\mathrm{O} 3$ de dezembro Evento On-line

auxiliando no entendimento. O protagonismo dessas ações de aprendizado deve-se aos estudantes.

Figura 7 - Desenvolvimento do problema 3.2 no ambiente MAPLE, parte III: sequência de gráficos de secante à tangente.

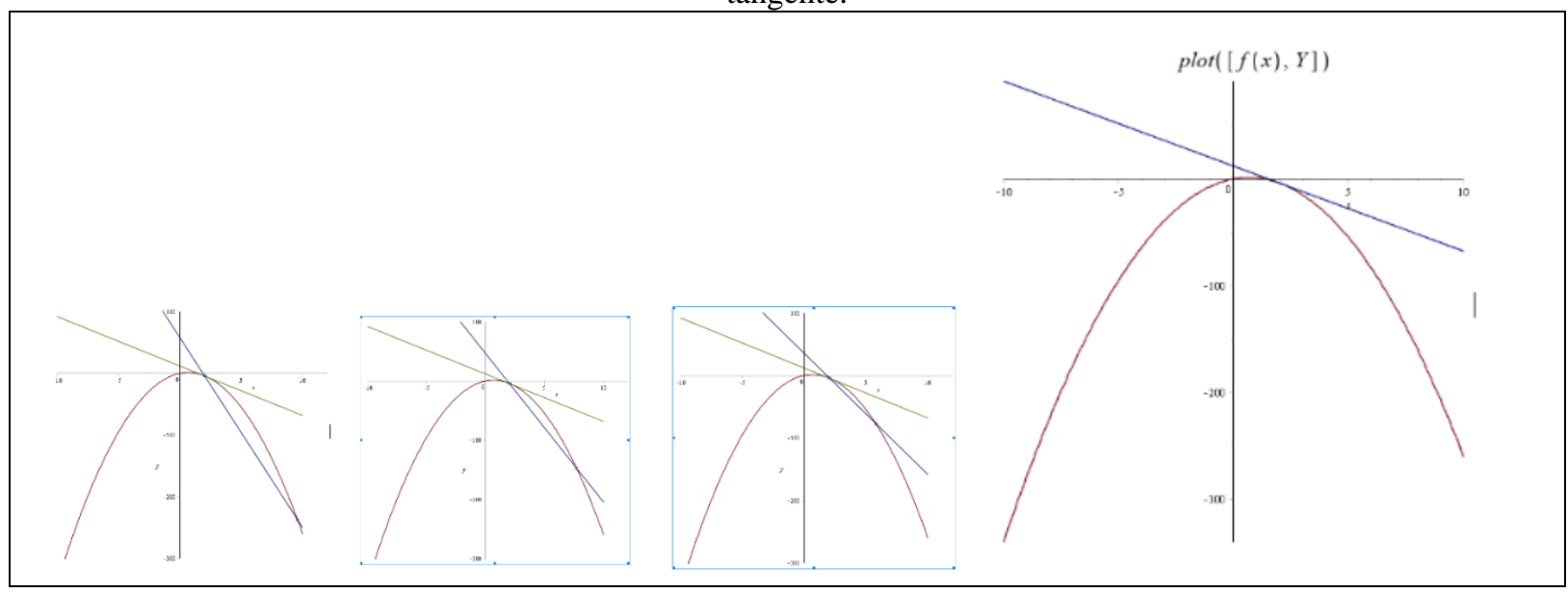

Fonte: Os autores

\subsection{Volume de revolução}

Nesta aplicação, há uma ligação muito interessante entre a matemática e a engenharia, permitindo a aplicação imediata do resultado encontrado em um objeto das engenharias. Sólidos de revolução são utilizados em estruturas de cascas, principalmente. A modelagem matemática desses casos, permite a geração do volume, permite o cálculo de volumes entre curvas (quantidade de material a ser empregado), composições de curvas de modelos simples, dentre outras possibilidades. Inicia-se pela definição do problema matemático do livro base (Figura $8)$.

Figura 8 - Definição do problema 3.3 no ambiente MAPLE.

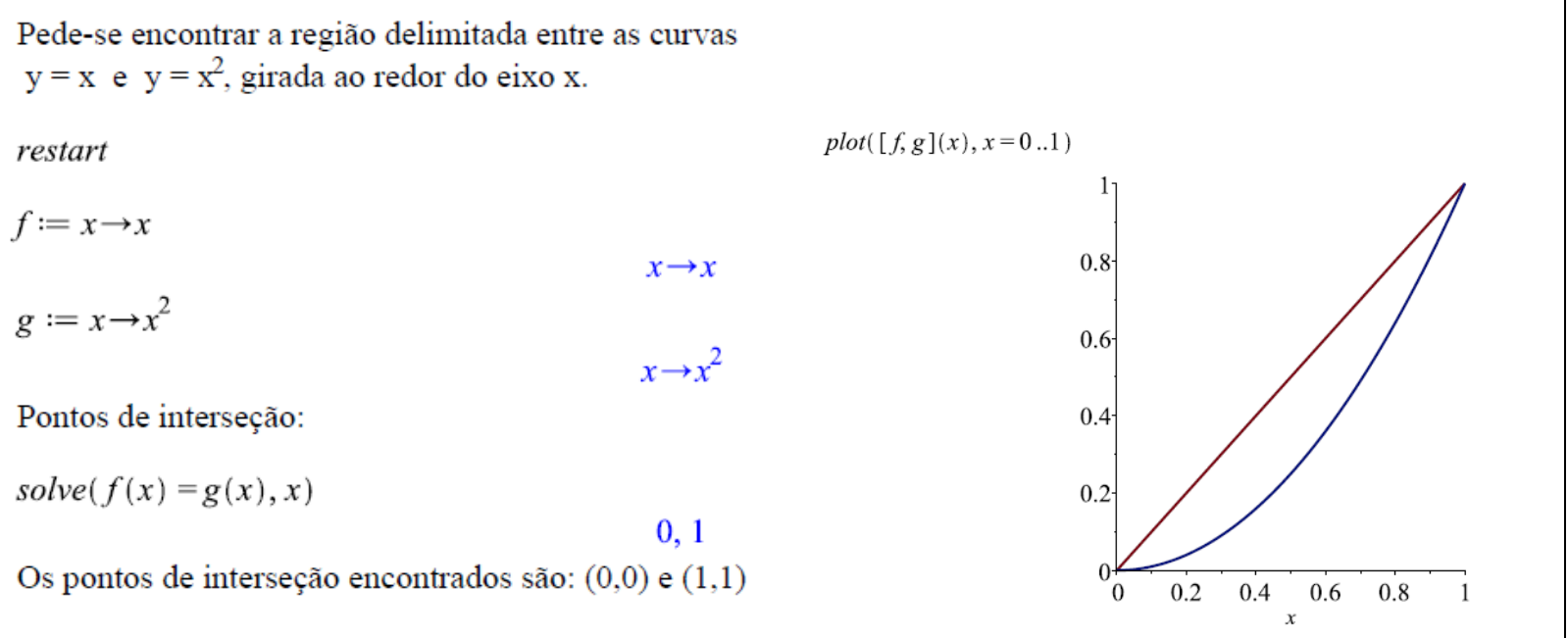

Fonte: Os autores 
A partir da curva (composta) geratriz do volume, procede-se o giro em torno do eixo especificado, gerando o volume requerido (Figura 9).

Figura 9 - Desenvolvimento do problema 3.3 no ambiente MAPLE: sólido de revolução requerido

$h:=x \rightarrow x-x^{2}$

O sólido de revolução resultante é:

$$
x \rightarrow x-x^{2}
$$

(1)

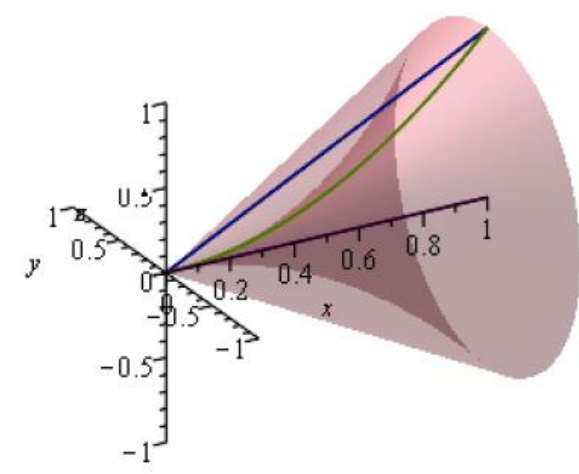

Fonte: Os autores

Finalizando o problema proposto, utilizam-se os conceitos envolvidos no cálculo integral para determinar o volume do sólido gerado por revolução de curvas (Figura 10). Em uma abstração, poderia representar o volume de concreto a ser utilizado em uma casca estrutural com a forma abordada.

Figura 10 - Desenvolvimento do problema 3.3 no ambiente MAPLE: cálculo do volume do sólido gerado. Construindo a integral para o volume do sólido, no intervalo de 0 a 1 :

$$
\begin{aligned}
& \int_{0}^{1} A(f(x)-g(x)) \mathrm{d} x=\int_{0}^{1} A(x)-A\left(x^{2}\right) \mathrm{d} x=\int_{0}^{1} \pi \cdot\left(x^{2}\right)-\pi \cdot\left(x^{2}\right)^{2} \mathrm{~d} x=\int_{0}^{1} \pi \cdot\left(x^{2}\right)-\pi \cdot\left(x^{4}\right) \mathrm{d} x=\int_{0}^{1} \pi \\
& \cdot\left[\left(x^{2}\right)-\left(x^{4}\right)\right] \mathrm{d} x ; \\
& \text { with(Student }[\text { Calculus } 1]) \text { : } \\
& \text { VolumeOfRevolution }(h(x), x=0 . .1 \text {, output }=\text { integral }) \\
& \qquad \int_{0}^{1} \pi x^{2}(x-1)^{2} \mathrm{~d} x
\end{aligned}
$$

VolumeOfRevolution $(h(x), x=0 . .1)$

$$
\frac{1}{30} \pi
$$

Fonte: Os autores 


\section{CONSIDERAÇÕES FINAIS}

O presente trabalho apresentou metodologia ativa híbrida, experiência realizada com alunos do curso de engenharia civil do Centro de Tecnologia da Universidade Federal de Alagoas. Foi particularizado para as disciplinas da matemática, especificamente os cálculos iniciais, nas quais se constatam quantidade excessiva de reprovações e abandonos nos semestres iniciais de cursos.

A experiência híbrida, aulas no modo clássico em sala e as tarefas extraclasse em metodologias ativas assistidas por plataforma computacional, mostrou-se adequada ao melhor entendimento, domínio dos conhecimentos matemáticos, a partir de cenários exploratórios de funções e suas manipulações matemáticas. As associações com problemas de engenharia, como modelagem necessária, trouxeram, também, motivação ao estudo da matemática superior.

A plataforma MAPLE, mostrou-se adequada para o estudo proposto, tendo em vista sua versatilidade na resolução dos problemas matemáticos, bem como a disponibilidade do passo a passo das operações, permitindo ao estudante a proatividade nas soluções e auxílio ao seu entendimento.

As metodologias ativas devem ser utilizadas nos cursos de engenharia civil após Novas DCN (Diretrizes Curriculares Nacionais), o que permitirá a possibilidade do engajamento motivado dos estudantes, como protagonistas do aprendizado, no ensino e na pesquisa nas disciplinas da matriz dos cursos.

\section{REFERÊNCIAS}

ARANGO, J., GAVIRIA, D., VALENCIA, A. Differential calculus teaching through virtual learning objects in the field of management sciences. Procedia - Social and Behavioral Sciences 176, 412 - 418, 2015.

FELIX, J. O., OLIVEIRA, T. E. de. Aprendizagem ativa em cálculo diferencial e integral na engenharia química. VI SICFEI - Simpósio de Iniciação Cientifica, Didática e de Ações Sociais de Extensão, São Paulo - SP. 2016.

ISTIANDARU, A., STYAWAN, F., HIDAYAT, A. S. E., ISTIHAPSARI, V. Learning differential calculus using self-regulated flipped classroom approach. Journal of Physics: Conference Series 1321, 022134. 2019.

JAAFAR, R., LIN, Y. Assessment for learning in the calculus classroom: a proactive approach to engage students in active learning. International Eletronic Journal of Mathematics Education, vol. 12, no. 3, pp. 503-520, 2017.

KLEIN, S. C. A. W., WEBLER, G., SANTI, C. B., KREMER, D. I. M. Estratégias metodológicas para o ensino de cálculo diferencial e integral nas engenharias. Anais VIII Congresso de Professores de Ensino Superior da Rede SINODAL. Joinville-SC, 2019.

OLIVEIRA, V. F. de (Org.). A engenharia e as novas DCNs: oportunidades para formar mais e melhores engenheiros. LTC Editora, Rio de Janeiro - RJ, 2019. 
PAULA, M. R. de, SOARES, G. A. A utilização de algumas ferramentas das metodologias ativas de aprendizagem para as aulas de cálculo diferencial. Anais do XII Encontro Nacional de Educação Matemática. São Paulo - SP, 2016.

SACHETTI, L. R. D. Análise da experiência de sala de aula invertida em curso de matemática básica para ingressantes em cursos de um centro de engenharias. Monografia de TCC de Especialização. UFSC. Joinville - SC, 2017.

TING, F. S. T., LAM, W. H., SHRO, R. H. Active learning via problem-based collaborative games in a large mathematics university course in Hong Kong. Educ. Sci., 9, 172, 2019.

\title{
ACTIVE LEARNING OF CALCULUS IN SOFTWARE-AIDED ENGINEERING
}

\begin{abstract}
The young people of the current world are one hundred percent connected to some kind of social network, with endless access to data and information instantly and everywhere. The arrival in university education brings expectations of updated forms of learning, at the speed that the generation usually moves. The crash in the early periods of engineering in which mathematics subjects, so important for the basis of courses, are given, and largely in the classic classroom way. This has resulted in high repetition and evasion rates. For some time, there have been more interesting methodologies to be used even in the basic cycles of engineering. In this context the present work is inserted, approaching the experience of active learning of calculus subjects, starting from the hybrid model, aided by a computerized platform. Civil Engineering students from the Technology Center of the Federal University of Alagoas were protagonists in active learning using the MAPLE platform in extra-class classes. The traditional classes took place and the development of the extra-class study was guided by question simulations, changing parameters and observing the change in behavior, besides a better understanding of functions and their mathematical manipulations. The association with effective engineering problems served as motivation for the in-depth learning of the calculation basis for the course student. Three cases studied in the present work are presented, under the guidance of an engineering professor for the direction of active methodology and consolidation in meetings to define strategies and clarify understandings. The conclusions were positive, as can be seen in the corresponding item of this work.
\end{abstract}

Keywords: Active Learning, MAPLE, Differential and Integral Calculus 